\title{
Investigation of silicon heterojunction solar cells by photoluminescence under DC-bias
}

\author{
Guillaume Courtois $^{1,2, a}$, Parsathi Chatterjee ${ }^{3,2}$, Veinardi Suendo ${ }^{4}$, Antoine Salomon ${ }^{1}$, and Pere Roca i Cabarrocas ${ }^{2}$ \\ 1 Total Énergies Nouvelles, La Défense, France \\ 2 LPICM, CNRS - École Polytechnique, Palaiseau, France \\ 3 Energy Research Unit, Indian Association for the Cultivation of Science, Kolkata, India \\ 4 Inorganic and Physical Chemistry Research Division, Institut Teknologi Bandung, Indonesia
}

Received: 30 July 2012 / Received in final form: 9 May 2013 / Accepted: 4 June 2013

Published online: 27 September 2013

(c) Courtois et al., published by EDP Sciences, 2013

\begin{abstract}
Photoluminescence measurements on solar cells are usually carried out under open-circuit conditions. We report here on an innovative approach, in which the samples are simultaneously illuminated and DC-biased, so that the luminescence can be monitored under several operating points, that is to say several injection levels, ranging from short-circuit conditions to the light-emitting regime of the device. The experiments were performed on in-house made c-Si/a-Si:H heterojunction solar cells illuminated by a continuous green laser diode and positively biased. The luminescence spectra obtained this way were compared to those obtained with no light excitation source, which corresponds to usual electroluminescence mode and dark $J(V)$. Firstly, the obtained luminescence spectra have shown the expected exponential dependence on the applied voltage. Furthermore, given that the amplitude of the emitted luminescence is proportional to the radiative recombination rate, this approach enables to indirectly characterise the nonradiative recombination phenomena. In the case of $\mathrm{HJ}$ solar cells with intrinsic thin layers processed on high quality FZ-wafers, non-radiative recombination is dominated by the defects at the c-Si/a-Si:H interface. The luminescence measurements presented here therefore give information on the quality of the surface passivation. An estimation of the interface defect density was achieved by comparing our experimental results with modelling.
\end{abstract}

\section{Introduction}

Among the different types of solar cells, crystalline silicon (c-Si)/hydrogenated amorphous silicon (a-Si:H) heterojunction (HJ) with intrinsic thin layer solar cells combine the advantages of crystalline cells, for instance high photocurrent, with those of thin film silicon solar cells: strong open-circuit voltage and low temperature coefficient. Moreover, given that the a-Si:H thin layers are deposited at low temperature, namely $200{ }^{\circ} \mathrm{C}$, by plasma processes, the thermal budget of this technology is low. Although HJ solar cells have been commercialised for fifteen years and a record cell efficiency of $24.7 \%$ has been reported ${ }^{1}$, there is still an issue regarding the properties of the c-Si/a-Si:H interfaces, which condition the solar cell performances [1]. More precisely, since the cells are processed on high quality monocrystalline silicon wafers, the

\footnotetext{
${ }^{a}$ e-mail: guillaume.courtois@polytechnique.edu

1 Panasonic, Press release, Feb. 12th, 2013
}

efficiency of the interface defect passivation is of crucial importance. As a matter of fact, the higher the interface defect density, the higher the surface recombination rate, and thus the poorer collection of photogenerated carriers. Surface recombination has been investigated through several techniques, such as photoconductance [2] or surface photovoltage [3]. In this paper, spectral luminescence will be emphasised.

In recent years, luminescence techniques have become more and more popular for the monitoring of defects in semi-conductor materials and solar cells. Since luminescence provides a fast and non-destructive assessment of radiative recombination, it turns out to be an indirect means for characterising non-radiative phenomena, which are for the cells in question, dominated by interface recombinations. Depending on whether the samples are optically or electrically biased, luminescence is referred to as either photoluminescence (PL) or electroluminescence (EL). Whereas PL constitutes a versatile technique, 
implementable from wafer to finalised devices, EL requires a finalised cell. Spatially resolved luminescence has been used to get mappings of various characteristics, from carrier lifetime to series resistance inhomogeneities [4]. PL measurements are generally carried out with the illuminated sample in open-circuit conditions, whereas EL is extracted from the light-emitting regime of a diode in the dark. When the optical power (respectively the electrical one) is increased, the amount of photogenerated (injected) carriers increases, hence an enhanced recombination - including radiative recombination - rate, which eventually enhances the luminescence signal.

In the case of a completed device, combination of both excitations is of course possible. This PL under DC-bias allows monitoring several operating modes of the device into question.

We report here on spectrally resolved EL and PL under DC-bias from HJ solar cells. The spectra obtained with and without light excitation have been compared; the experimental results have then been compared to modelgenerated ones. An estimation of the actual interface defect density $\left(N_{i d}\right)$ of our samples could be extracted by modelling the experimental light $J(V)$ characteristics.

\section{Experimental procedure}

The experiments were carried out on $2 \times 2 \mathrm{~cm}^{2}$ inhouse made heterojunction solar cells processed on doubleside polished, float zone, $n$-type, $280 \mu$ m-thick monocrystalline silicon wafers whose resistivity was of $3 \mathrm{Ohm} \mathrm{cm}$. Once the wafers had undergone a hydrofluoric acid dip, thin $(5 \mathrm{~nm})$ intrinsic layers ensuring the interface passivation as well as $15 \mathrm{~nm}$-thick doped a-Si:H layers constituting the front emitter and the back surface field were deposited by radio-frequency plasma enhanced chemical vapour deposition (RF-PECVD) at $200{ }^{\circ} \mathrm{C}$ [5]. Indium tin oxide (ITO) layers were then deposited by magnetron sputtering on both front and rear sides as anti-reflective coating and electrodes. Finally, the front contact grid and the full sheet back reflector were both made of evaporated silver (Fig. 1). In order to check the general behaviour of the cells, $J(V)$ characteristics were measured under 1 sun on a AAA class solar simulator. Efficiencies of more than $15 \%$ were assessed, associated with $V_{o c}, J_{s c}$ and fill factor of $690 \mathrm{mV}, 31 \mathrm{~mA} / \mathrm{cm}^{2}$ and $73 \%$, respectively.

Coming to the luminescence investigations, the photoexcitation was performed using a continuous laser diode delivering an optical power of $15 \mathrm{~mW}$ at $532 \mathrm{~nm}$, wavelength at which the reflectivity of the sample displayed a minimum. The spotlight section was around $5 \mathrm{~mm}^{2}$, which corresponds to an impinging flux on the sample of around $10^{18}$ photons $\mathrm{cm}^{-2} \mathrm{~s}^{-1}$. A SourceMeter ${ }^{\circledR}$ by Keithley was used simultaneously as DC-supply and ammeter, in order to DC-bias the samples and at the same time acquire $J(V)$ curves. All measurements were carried out at room temperature. As for the detection part of the setup, the collected luminescence signal transited via an optical fibre to a grating spectrometer by Horiba and finally reached a

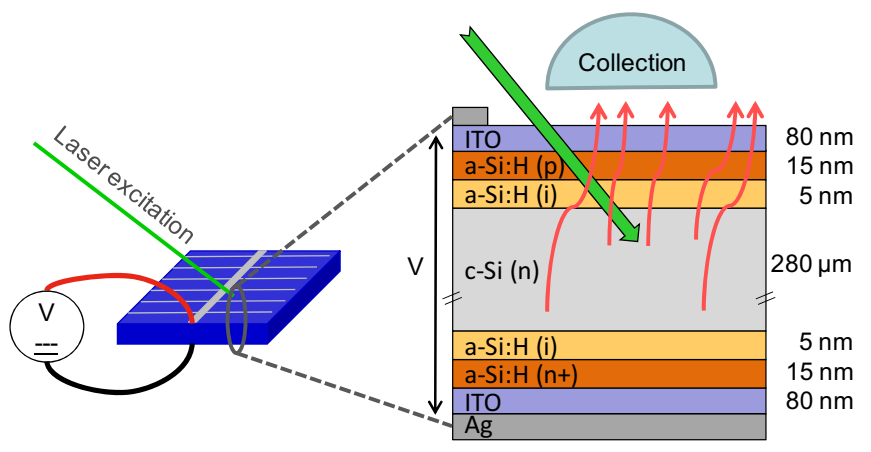

Fig. 1. Schematic of a HJ solar cell in our experimental configuration, showing the DC-supply, as well as the optical excitation supplied by a green laser.

mono-crystal InGaAs detector cooled down by liquid nitrogen. Over a set of measurements, the sample and the optical fibre remained fixed, so that all luminescence spectra were acquired from the same area on the device, coinciding with the illuminated area when the laser diode was turned on (Fig. 1).

\section{Simulation model}

The one-dimensional electrical-optical model ASDMP (Amorphous Semiconductor Device Modelling Program) $[6,7]$, that has recently been extended to equally model HJ solar cells [8], solves the Poisson's equation and the two carrier continuity equations under steady state conditions for the given device structure. The complex refractive indices for each layer of the structure are required as input and have been measured in-house by spectroscopic ellipsometry.

The emitted photon flux density from $n$-type c-Si wafers can be described by the generalized Planck's/ Kirchoff's law, which takes into account non-homogeneous carrier distribution and hence a non-constant quasi-Fermi level splitting, as well as a non-homogeneous generation profile and reabsorption of luminescence photons, and reads as follows [9]:

$$
\begin{aligned}
& d \Phi_{\text {lum }}(\hbar \omega)=\frac{(\hbar \omega)^{2}}{4 \pi^{2} \hbar^{3} c^{2}} \exp \left(\frac{-\hbar \omega}{k T}\right) \\
& \quad \times\left[\int_{0}^{\mathbf{t}} A(\hbar \omega, x) \exp \left(\frac{E_{F n}(x)-E_{F p}(x)}{k T}\right) d x\right] d \hbar \omega,
\end{aligned}
$$

where $t$ is the thickness of the wafer. $A(\omega, x)$, the absorptivity of light of a particular energy $(\hbar \omega)$ at a given position inside the wafer, and the quasi-Fermi level splitting $E_{F n}(x)-E_{F p}(x)$ inside it can be calculated by model ASDMP. Details of the procedure are given in reference [9], where ASDMP has been implemented to the simulation of luminescence in heterostructures.

For a good luminescent diode (i.e., in which the amorphous/crystalline interface defect density $\left(N_{i d}\right)$ is less than $\left.\sim 5 \times 10^{11} \mathrm{~cm}^{-2}\right), E_{F n}-E_{F p}$ is constant and equal to $q V$, 
Guillaume Courtois et al.: Investigation of silicon heterojunction solar cells by photoluminescence under DC-bias

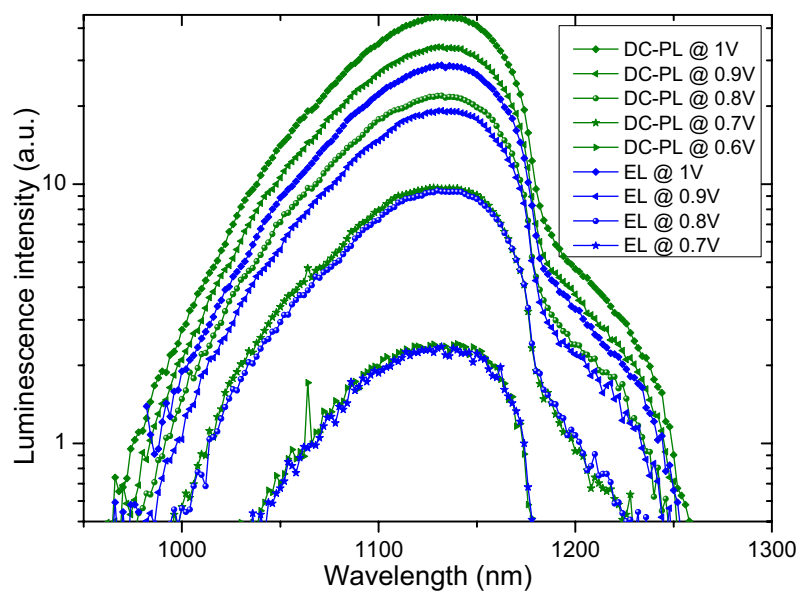

Fig. 2. Experimental EL and PL under DC-bias spectra.

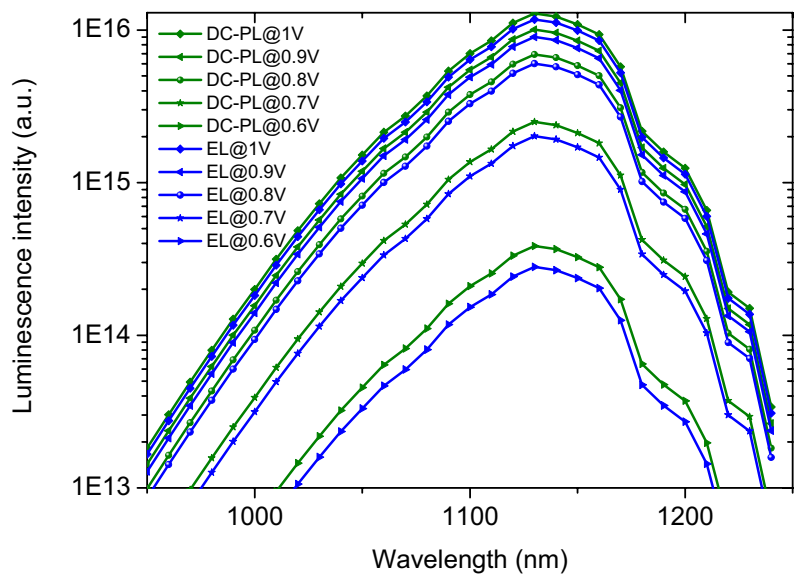

Fig. 3. Model-generated EL and PL under DC-bias spectra.

where $V$ is the applied voltage. Alternatively it is equal to $q V_{o c}$, when, as is generally the case, the $\mathrm{PL}$ is measured under open-circuit conditions. Model-generated EL and PL under DC-bias results are presented in Figures 3 and $4 \mathrm{~b}$ and will be discussed in Section 5 .

\section{Experimental results}

EL spectra were obtained under applied voltages up to $1 \mathrm{~V}$, at the same time as dark $J(V)$ curves. By turning the laser on, everything else kept constant, both light and DC-bias excitations were combined, hence a luminescence mode referred to as PL under DC-bias.

Experimental luminescence spectra are displayed on Figure 2. On all spectra, the maximal luminescence intensity occurs at $1130 \mathrm{~nm}$, which corresponds to band-toband radiative recombination in c-Si. If the amorphous silicon were to contribute to the luminescence, it would emit below $950 \mathrm{~nm}$, where no luminescence is detected. As a matter of fact, the a-Si:H layers are too thin to contribute to the luminescence signal. As to the shoulder appearing on the high wavelength side, it has been accounted for by recombination bringing into play the creation of two phonons [10].
Obviously, both EL and PL under DC-bias intensities increase with the applied voltage (see Eq. (1)). For a given applied voltage, PL under DC-bias turns out to be more intense than pure EL, which originates in the superposition of photogenerated carriers to the injected ones. Moreover, when moving from EL to PL under DC-bias, the shape of the spectra remains unaltered; the total integrated flux, corresponding to the luminescence yield, is therefore proportional to the peak intensity. That being stated, we will henceforth go on dealing with the values of the peak intensity when comparing the measurements one to another.

The variation of the peak intensity with the applied voltage is presented on Figure 4a for both EL and PL under DC-bias modes. Two regimes are underlined: at low applied voltages, that is to say below $0.7 \mathrm{~V}$, both curves exhibit a diode behaviour, the luminescence intensity increasing exponentially with the applied voltage, whereas for higher voltages a kind of saturation occurs, associated with a slower increase in the luminescence intensities. A surprising feature is the discrepancy between the ideality factors extracted from the $0.5-0.7 \mathrm{~V}$ range of applied voltage, which turn out to be of 1.3 and 2.2 for EL and DC-PL, respectively, whilst a similar dependence upon the applied voltage was expected. The mismatch observed experimentally probably stems from lateral effects due to the competition between the illuminated and the shaded areas. Even more surprising from our point of view is the third value of the ideality factor obtained from $J_{\text {dark }}$, which turned out to be of 1.7, so between the previous ones. Note that, for applied voltages around $0.6 \mathrm{~V}$, a single-diode model suffices to render the dominating current transport [11].

\section{Discussion and comparison with modelling}

In order to be sure that our experimental results were not affected by a possible change in temperature induced by either the DC-bias up to $1 \mathrm{~V}$ - which corresponds to an electrical power of $350 \mathrm{~mW}$ - or the impinging optical power, we had a closer look at the high energy region, namely above $1.2 \mathrm{eV}$ (i.e. below $1000 \mathrm{~nm}$ ). The expression for absorptance $A$ is given in equation (2) [12]. In equation (1) used in ASDMP, multiple reflections are considered until all the long wavelength luminescent photons are either reabsorbed in the c-Si wafer, or lost by absorption in the rear contact or in the non-luminescent front layers, or by reflection at the front. Equation (2) involves frontside and back-side reflectivities $\left(R_{f}\right.$ and $R_{b}$, respectively), as well as the absorption coefficient in crystalline silicon $(\alpha)$. All these quantities are spectrally dependent. $t$ still stands for the thickness of the wafers, namely $280 \mu \mathrm{m}$.

$$
\begin{aligned}
A(\hbar \omega)= & \frac{\left(1-R_{f}\right)\left[1-R_{b} e^{-2 \alpha t}-\left(1-R_{b}\right) e^{-\alpha t}\right]}{1-R_{f} R_{b} e^{-2 \alpha t}} \\
& \underset{\hbar \omega \geqslant 1.2 \mathrm{eV}}{\longrightarrow} 1-R_{f} .
\end{aligned}
$$

The more energetic the radiation, the higher the value of $\alpha$. Namely, for photons, $\alpha$ is higher than $10 \mathrm{~cm}^{-1}$ [13], 
EPJ Photovoltaics

Table 1. Solar cell output parameters and the diode ideality factor of the DC-PL peak tion intensity versus $V$ curve, the EL peak intensity versus $V$ curve and the dark $J(V)$ characteristic of the deposited HJ solar cell on $n$-type wafer, compared to modelling results.

\begin{tabular}{cccccccc}
\hline & $J_{s c}$ & $V_{o c}$ & \multirow{2}{*}{$F F$} & Efficiency & \multicolumn{3}{c}{ Diode ideality factor, $n$} \\
\cline { 6 - 8 } & $\left(\mathrm{mA} \mathrm{cm}^{-2}\right)$ & $(\mathrm{V})$ & & $(\%)$ & DC-PL peak & EL peak & $J_{\text {dark }}$ \\
\hline Experiment & 31.0 & 0.692 & 0.73 & 15.5 & 2.2 & 1.3 & 1.7 \\
Model & 31.36 & 0.691 & 0.77 & 16.8 & 1.06 & 1.04 & 1.14 \\
\hline
\end{tabular}

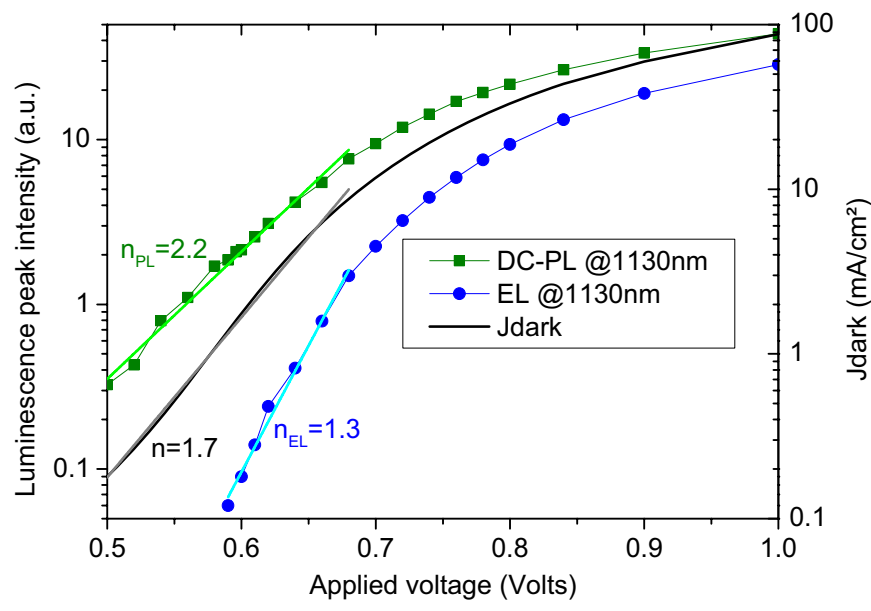

(a)

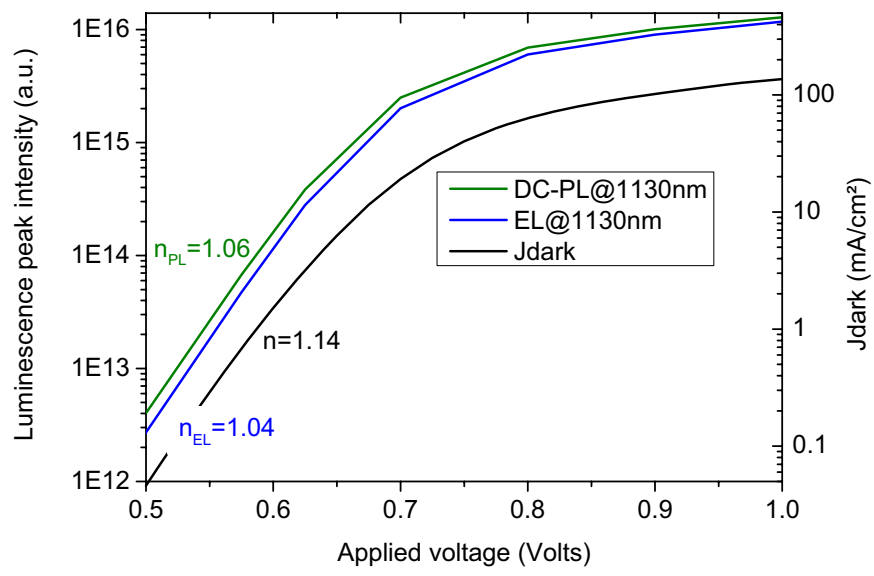

(b)

Fig. 4. Variations of (a) measured and (b) model-generated EL and PL peak intensities under DC-bias with respect to the applied voltage. Comparison with (a) the measured and (b) the modelled dark $J(V)$ characteristic.

therefore $2 \alpha t$ is greater than 5 and the expression of $A$ simplifies to $\left(1-R_{f}\right)\left[1-\left(1-R_{b}\right) e^{-\alpha t}\right]$. Now, $R_{b}$ has been evaluated to $93 \%$ over the $900-1000 \mathrm{~nm}$ range, thus $A$ reasonably reduces to $1-R_{f}$, which varies very little from $80 \%$ for the considered a-Si:H/ITO stack deposited on polished c-Si. Subsequently, above $1.2 \mathrm{eV}$, the spectral dependence of $\mathrm{d} \Phi_{\text {lum }}$ becomes restrained in the product $(\hbar \omega)^{2} \exp \left(\frac{-\hbar \omega}{k T}\right)$. Consequently, it reads (Eq. (6)):

$$
d \log \left(\Phi_{l u m}(\hbar \omega)\right)=\left(2 \log (\hbar \omega)-\frac{\hbar \omega}{k T}+C\right) d \hbar \omega
$$

where $C$ accounts for the spectrally independent quantities. Considering energies and temperatures of the order of $1 \mathrm{eV}$ and $300 \mathrm{~K}$, respectively, the term $\log (\hbar \omega)$ in the above expression becomes negligible, and thus the slope of the high-energy tail of the luminescence peak, when plotted in logarithmic scale, is simply $-1 / k T$. It is clear from Figure 2 that the experimental luminescence spectra all show the same slope below $1000 \mathrm{~nm}$, which attests to the absence of significant temperature change over the experiments.

Modelling of the illuminated $J(V)$ characteristics under AM 1.5 light of the structure shown in Figure 1 was carried out in order to get an idea of the defect density at the a-Si:H/c-Si interfaces. It has been previously found [9] that when $N_{i d}$ exceeds $10^{12} \mathrm{~cm}^{-2}$ at the emitter/c-Si interface, the PL intensity is too weak for accurate measurement. Regarding the material properties, a doping density of $2 \times 10^{15} \mathrm{~cm}^{-3}$ was assumed in the $n$-doped wafer, corresponding to a resistivity of $3 \mathrm{Ohm} \mathrm{cm}$. The other input parameters are also similar to those given in reference [9]. A comparison to the experimental measurements is shown in Table 1. Luminescence features were also modelled under voltage bias, while for PL excitation, additional generation by a $530 \mathrm{~nm}$ light was considered in parallel to the voltage excitation.

The HJ cell $V_{o c}$ is known to be strongly sensitive to the defect density at the emitter/c-Si interface $[8,9]$ and this fact has been used to estimate $N_{i d}$ at this junction from modelling and turns out to be $4.5 \times 10^{11} \mathrm{~cm}^{-2}$. Photovoltaic performance is almost insensitive to the c-Si/BSF $N_{i d}$ below $5 \times 10^{11} \mathrm{~cm}^{-2}[8,9,14]$ and a value of $10^{11} \mathrm{~cm}^{-2}$ has been assumed here in the modelling calculations. However, the calculated FF does not agree with the measured value, because of the high series resistance of the contacts, estimated to be $\sim 3 \mathrm{Ohm}$. The calculated value of the FF would correspond to the measured value corrected for the series resistance of the contacts. Also compared in the same table are the diode ideality factors for the dark $J(V)$ characteristics, and the PL under DC-bias and EL peak intensities (at $\lambda=1130 \mathrm{~nm}$ ).

The calculated PL and EL curves under applied voltages are plotted in Figure 3. The first satisfactory feature is that they are of the same shape as the experimental ones (see Fig. 2). In addition, the variations of EL and PL under DC-bias peak intensities with respect to the applied voltage, as well as $J_{\text {dark }}$ (Fig. 4), show, like in the experimental curves, a break of slope at $0.7 \mathrm{~V}$. Indeed, above this value, the electrical properties of the device are dominated by the resistive effects. Nevertheless, the ideality factors extracted from the $0.5-0.7 \mathrm{~V}$ region of 
these curves proved to be of 1.06, 1.04 and 1.14 for PL under DC-bias, EL and $J_{\text {dark, }}$, respectively, so very close to each other, which was expected. Indeed, EL yield and $J_{\text {dark }}$ are supposed to display the same dependence upon the applied voltage. Moreover, assuming the EL yield is proportional to $\exp \left(\frac{q V}{n k T}\right)$, one would expect for the PL yield under DC-bias: $\phi_{D C P L} \propto \exp \left(\frac{q(V+\Delta V)}{n k T}\right)$ where $\Delta V$ accounts for the extra junction voltage conveying the enhanced splitting of the quasi-Fermi levels induced by the laser excitation. As a result, when plotted in logarithmic scale, a simple shift is expected between the $\operatorname{EL}(V)$ and DC-PL $(V)$ curves, as displayed in Figure 3.

The ideality factors obtained from modelling turn out to differ significantly from the experimental ones. As a matter of fact, a homogeneous illumination on the entire device is taken into account in the one-dimensional model, whereas strong lateral effects occur in our experimental configuration.

\section{Conclusion and perspectives}

By combining optical and electrical excitations, luminescence spectra from in-house elaborated heterojunction with intrinsic thin layer solar cells were obtained at many operating points. As expected on this kind of devices processed on high quality monocrystalline wafers, whose properties are limited by the interface features, EL and PL under DC-bias spectra proved to be similar in shape, conveying regular carrier distributions within the wafer. In both cases, the luminescence yields exhibited an exponential dependence with the applied voltage. However, the ideality factors calculated from EL, PL under DC-bias and dark $J(V)$ curve significantly differed from each other and were far from unity. This feature may be due to lateral resistive losses which would alter the local diode voltage in some fashion, all the more so as only the investigated fraction of the cell was illuminated. Next set of experiments will be performed on fully illuminated cells. Our experimental results were compared to 1D ASDMP modelling, allowing an estimation of the front interface defect density, which proved to be of $\sim 4.5 \times 10^{11} \mathrm{~cm}^{-2}$. Experiments and modelling agreed on the detrimental effect of series resistance at voltages higher than $0.7 \mathrm{~V}$.
We intend to extend this study to samples processed on textured wafers and we expect, that, in conjunction with photoconductance measurements, this study will help discriminate between field effect and chemical passivation, the latter being less sensitive to the injection level.

This work was performed within the joint solar R\&D team between Total and LPICM.

\section{References}

1. S. De Wolf, A. Descoeudres, Z.C. Holman, C. Ballif, Green 2, 7 (2012)

2. M. Garin, U. Rau, W. Brendle, I.A.R. Martin, J. Appl. Phys. 98, 093711 (2005)

3. L. Korte, A. Laades, K. Lauer, R. Stangl, D. Schaffarzik, M. Schmidt, Thin Solid Films 517, 6396 (2009)

4. T. Trupke et al., in Proceeding of the 22nd EPVSC, Milan, 2007

5. P. Roca i Cabarrocas, J.B. Chévrier, J. Huc, A. Lloret, J.Y. Parey, J.P.M. Schmitt, J. Vac. Sci. Technol. A 9, 2331 (1991)

6. P. Chatterjee, J. Appl. Phys. 76, 1301 (1994)

7. P. Chatterjee, J. Appl. Phys. 79, 7339 (1996)

8. M. Rahmouni, A. Datta, P. Chatterjee, J. Damon-Lacoste, C. Ballif, P. Roca i Cabarrocas, J. Appl. Phys. 107, 054521 (2010)

9. A. Datta, M.-H. Song, J. Wang, M. Labrune, S. Chakroborty, P. Roca i Cabarrocas, P. Chatterjee, J. NonCryst. Solids 358, 2241 (2012)

10. G. Weiser, S. Kazitsyna-Baranovski, R. Stangl, J Mater. Sci.: Mater. Electron. 18, 93 (2007)

11. T.F. Schulze, L. Korte, E. Conrad, M. Schmidt, B. Rech, J. Appl. Phys. 107, 023711 (2010)

12. R. Brüggemann, in Physics and Technology of AmorphousCrystalline Heterostructures Silicon Solar Cells, edited by W.G.J.H.M. van Sark, L. Korte, F. Roca (Springer-Verlag, Berlin, Heidelberg, 2012)

13. M.A. Green, M.J. Keevers, Prog. Photovolt.: Res. Appl. 3, 189 (1995)

14. S. de Wolf, in Physics and Technology of AmorphousCrystalline Heterostructures Silicon Solar Cells, edited by W.G.J.H.M. van Sark, L. Korte, F. Roca (Springer-Verlag, Berlin, Heidelberg, 2012) 